\title{
An Approach of an Idea Management Platform to Improve the Innovation Process
}

\author{
Pedro Marcelo \\ Centre of Innovation and \\ Development, ISPGaya \\ Av. dos Descobrimentos, 333 \\ 4400-201 V.N.Gaia, Portugal
}

\author{
José Monteiro \\ Faculty of Engineering, Univ. of \\ Porto \& INESC TEC \\ Rua Dr. Roberto Frias, $\mathrm{s} / \mathrm{n}$ \\ 4200-465 Porto, Portugal
}

\author{
Fernando Almeida \\ Faculty of Engineering, Univ. \\ Porto \& INESC TEC \\ Rua Dr. Roberto Frias, $\mathrm{s} / \mathrm{n}$ \\ 4200-465 Porto, Portugal
}

\begin{abstract}
While innovation and creativity are critical to an organization's success, it cannot be forced or even created only by top company executives or dedicated innovation teams. Instead, the appropriate environment and processes need to be crafted, so that an employee's innate creativity is naturally forthcoming.
\end{abstract}

This paper presents a project that was developed in order to promote the human capital of an entity or organization through individual dissemination of ideas whose context is to improve processes, reduce costs, increase efficiency or to simplify administration and bureaucracy.

The platform provides to all employees of an organization the opportunity to express their ideas publicly. The ideas are anonymously approved a priori and the adopted voting process follows the methodology of Likert.

\section{General Terms}

Information Systems Applications, Web Applications, Database Design and Models.

\section{Keywords}

Innovation, Ideas, Likert, Collaboration, Web Platform.

\section{INTRODUCTION}

Organizations increasingly value contributions from workers, customers, partners, among others in the generation of new ideas to improve their competiveness. Several methods have been used to generate consensus to select ideas and improve their implementations. Approaches based on information technologies that intends help in the process of idea generation have increased in relevance, namely in the mediation of users interaction. These approaches intend mostly to avoid organizational or human inhibitors. Web based solutions combine three necessary factors for mediation based on computer systems to be successful among idea contributors: (i) they are cross-platform; (ii) are available anytime and anywhere; (iii) allow anonymity of the contributors.

The efforts to make people contribute with new ideas are not recent. Several techniques and methods have been used. Depending on the context, some methods seem to be more efficient than others. The paradigm is how to get the best of each method in order to increase the rating of new ideas. The system presented in this paper have four main objectives: (i) to be simpler as possible; (ii) to be available anytime and anywhere; (iii) to allow contributors anonymity; (iv) to help to increase idea generation rate.

The system offers a minimum set of users' category to avoid parallelism among system hierarchy and organization hierarchy: one system administrator; one or more managers; one or many users. The organizational role of a certain person is not relevant to define its role as a user. Each user is free to suggest any idea without fearing "retaliation" by other users. The same is applicable when users rate the submitted ideas.

The document is organized with the following sections: in section two are presented a literature review about ideas and innovation; in the section three are presented similar projects; in the section four the system is described; in section five a discussion of the results is presented; and finally some conclusions are drawn.

\section{LITERATURE REVIEW}

\subsection{Relation between Ideas and Innovation}

The interaction between individuals plays a critical role in the development of new ideas. A community of users interacting contributes to the amplification and the creation of new knowledge [1]. The organization supports creative individuals or provides a context to create knowledge. A study presented by Lindsey [2] reveals that individuals obtain significant number of ideas from their teammates. Also, they argue that a systematic exchange of a set of ideas is more productive than having all ideas displayed to all participants. This means that if a person views only a subset of ideas (for example: ideas of one's own team) in a given moment, one tends to be more focused on a certain domain and, in consequence, more creative than if one can continuously view the ideas from all participants [2].

Having ideas is known as part of human nature. During their life, people have millions of ideas. As history is considered important to understand the future, past experiences are crucial to influence people's understanding of new ideas. To evaluate an idea it is important to consider some factors like past experience, the notion, the concept and reach consensus [3]. Assuming these factors as facts, it seems logical that people which have different experiences during their life, have different conceptions about ideas. However, evidence points to ideas being seen as a new discovery, an innovative process or even a field of opportunity [4]. In business world, ideas sound as hope to differentiate from competitors. 
In the context of business organizations, while ideas prepossess to make different from competitors, innovation goes far and, are implies the needs of economic performance and the value creation to get competitive. It results that Innovation processes have necessarily impact on the costs of a final product. The ability to innovate is the core of the engineering/inventing a new product and it is constrained by the four pillars of innovation: leadership, culture \& climates, people and structures $\&$ processes [2].

Nonaka [1] argues that ideas are formed in the mind of individual, considering that ideas are vision driven. To have an idea does not mean that it will materialize [5]. A first approach is trying to understand how ideas are related with creativity and how both fit in the context of innovation. Creativity and innovation are linked by ideas. According to Gurteen [5], creativity is the process of generating ideas and innovation is the process of shifting ideas and how they are implemented. In summary, new ideas are the product that result of the creativity, while innovation is about to put new ideas in action.

\subsection{The Process of Evaluating Ideas}

The interest on idea generation (IG) techniques is not new in the organization environments. Since over half of the past century, several structured IG methods have been developed to stimulate creativity, both for individuals and for groups [67]. Such methods help to stimulate the human mind with different techniques and regarding different purposes. For example, the overload of information is the opposite of the information absence. However, both situations are considered as inhibitors to the generation of new ideas. For each situation, specialists argue that different methods should be used.

With the "democratization" of the use of the Web and the computerized devices, associated to the mobility that it provides today, has emerged the conscience that the computers can be a useful tool to support IG [8]. Computer machines play an important role in information storage, making complex calculus, or simply remembering tasks. Currently, people have to deal with a so large amount of information that is typically beyond people's physical capabilities. The computer "assistance" allow people to support more activities than they are physically capable. It is feasible to think that computers may play an important role in the support of IG, in particular on innovation processes.

A common sense view allows us to think that IG, from a computer perspective view, it is a mathematical problem, and from a human perspective view, it is a cognitive and sociological problem. Goguen [9] argues that it is important to have good mathematical foundations in addition to a broad understanding of the problem supported by cognitive psychology and sociology models. Concerning the understanding of how humans generate ideas, Shah et al [7], proposes taxonomy of formal methods for IG [6]. It distinguishes two main classes of methods: (i) Intuitive methods: work by stimulating the unconscious thought processes of the human mind. Given the unpredictability of the human mind it may be more complicated to implement these methods, component by component, in a computer machine. However, it helps to an understanding of how humans deal with the problem. (ii) Logical or rational methods involve systematic decomposition and analysis of the problem. These methods make use of science and engineering principles and/or catalogs of solutions, and are algorithmic oriented.
According to Jogalekar \& Mangla [8], logic of permutation and combination can be applied to a machine for return of an output, along with the ability to perform the task of selection via trained networks, which learn and select laterally the correct input to give a final selected output. Authors establish an analogy between a human brain and computer machines to justify the creation of an algorithm that helps a machine in the generation of its own set of ideas. Furthermore, the algorithm is supposed to use a trained network to generate the final idea [8]. A limitation of this approach is to consider only one method of IG (lateral think method) to justify its appliance. Also, the use of a transformational method (used to generate ideas by modifying existing ideas) may reveal itself to be less appropriate when there are no ideas.

A system named GUNGEN-Spiral was developed from Munemori et. al [10], for collecting ideas, saving ideas, sharing ideas, shaping ideas and reusing ideas. Authors argue that if was physically possible to collect ideas anytime and anywhere, then a large quantity of high quality ideas would be collected. To put in practice they have developed a system which runs on palm devices based on KJ-Method. It consists on: (i) Transmitting data from a palm device to a PC, classifying data and adding keywords; (ii) Members can read the shared data by using a browser; (iii) If a new idea comes from others it can be written as a new idea; (iv) Participants can use collected data or just input their own ideas; (v) A result can be returned after using the KJ-Method. Members can refer and examine the result of the KJ-Method [10]. On 2003 was presented a refined version of the system called GUNGEN DX II [11]. The use of a germinal method identifies the system as appropriate to be used when there are no existing previous ideas. Some limitations can be identified on this system: The large spectrum of ideas that can be submitted and the absence of a domain can compromise the objectivity of the results in some cases.

A critical view about the mentioned systems on this section reveals that each system only explored one formal method for IG. Also, the behavior of each one cannot be compared based on the chosen methods. Also, idea evaluation processes embodies high subjectivity.

\subsection{Collaborative Innovation Process}

Since the beginning of this century several networks have formed over the Web and new forms of socialization and collaboration have emerged. The concept of collaborative networks $(\mathrm{CN})$ has brought the attention of the academic community, initially to characterize them, to investigate what they do, and to determine their value to organizations. According to Camarinha-Matos \& Afsarmanesh [12] a CN is a network that involves autonomous organizations and people in a distributed and heterogeneous context, interacting and collaborating to achieve common goals, mediated by information technologies [12]. To Carneiro et al. [13], if those interactions are part of the inter-organizational relationships, then the $\mathrm{CN}$ is a collaborative network of organizations (CNO). A new contribution in this field, called Epistemic Networks (EN), has been proposed by Roth [14]. The concept of EN is supported by a framework based on three complimentary network types: (i) a social network: a structure formed by agents (e.g., individuals) and (or) community agents (e.g., organizations) linked by mutual interests; (ii) a semantic network: an organized structure that represents concepts and the relationships between them; (iii) and a sociosemantic network: an organized structure that links individuals and communities interests with the concepts [14]. These achievements have been explored by Pereira et al. [15] 
and applied to $\mathrm{CNO}$. Considering a $\mathrm{CN}$, in order to represent people and organization interactions, and its conceptual structures, a CN can be faced as a form of EN. As argued by Pereira [16], a $\mathrm{CN}$ is composed by a social-network, a semantic network, and a socio-semantics network. It is important to refer that socio-semantics can be characterized as an instrument of knowledge and information share in the $\mathrm{CN}$ context [16]. In the present subject socio-semantics represents the knowledge about the network.

Nonaka [1] conceives innovation as a process in which an organization creates and defines problems, and actively generates knowledge to solve them. In such scenario, where innovation is characterized as an interactive process involving collaborative relationships, interactions, and exchange of knowledge [17], the CNO may provide the appropriate conditions for innovation. Regarding the needs of organizations to be creative and innovative, a CNO that provides conditions to innovation can be named as an Innovation Network (IN) [18].

Different studies have been done in innovation networks addressing ideas, knowledge transfer, among others. A limitation to this work is the lack of studies in socio-semantic networks. However, some contributions in social network analyses can be identified on the following cases. Björk \& Magnusson [19] argues that there is an interrelationship between the network connectivity and the quality of the innovation ideas created. Another empirical study on the analysis of the impact of network structure on knowledge transfer revealed that information and knowledge is more accurately transmitted in networks where many actors are directly connected to each other [20]. Like in the first case, the connectivity of the network is an influent factor. In the analysis of a community/network of innovation, the size (number of members) and time (moments of evaluation) are the basic properties to examine the evolution of the network and the behavior of its members [17]. Nevertheless, Hautz et al [17], also consider important to identify user types. With this approach it is intended to get a better understanding about the role of participants and their influence on the innovative context. Regarding the research objectives, these two contributions help to demonstrate that the network influences the process of the generation of new ideas.

\section{SIMILAR PROJECTS}

Similar projects were researched in order to identify relevant features and concepts. Due to the growing number of applications to manage ideas founded, this study selected three examples to provide a better contextualization: (i) Crowdicity $^{1}$; (ii) IdeasMine ${ }^{2}$; (iii) Ideacomb ${ }^{3}$.

Crowdicity and Ideacomb are very complete in terms of features and integration with social networks and focused in the increase of idea generation ratio. These applications also include the possibility of users interacting with different devices and (or) with different operating systems. The concept of these applications is based on software on demand, which makes them available anytime and anywhere. Despite the announcements of each software vendor, some common features where identified: idea capturing; customers, partners involvement; adoption of Key Metrics of 'Open Innovation' initiative; collaborative environment; communities building; detection of similar ideas; report generation; search possibilities. The different type of actors that can be involved and the cross situations to be managed can make the administration process very complex to small organizations. Different from these two applications, IdeasMine, is simpler and more focused on idea improvement. The application is Web based, but its utilization is more oriented to manage idea processes inside the organization. The interaction is based in three main types of actors: administrator of platform, manager and contributors. The idea lifecycle has four basic steps: idea posting; studding idea; idea on implementation; and idea implemented. Comparing with Crowdicity and Ideacomb, IdeasMine seems to be less complicated for small organizations and when a discussion is needed; its process is faster and more assertive.

IdeasMine is closer to the concept presented within this study rather than Crowdicity and Ideacomb.

\section{APPROACH}

The project establishes interaction with three organizational players that will have different permission levels on the system:

- Administrator - has access to all functionalities offered by the system. It can also customize the role of other users;

- Moderator - can create, approve, search and vote in ideas. Additionally, he can also create and search for users;

- Collaborator - can create, search, and vote for ideas.

There is, however, the possibility for creation of new types of actor, which gives enough flexibility to the application to adjust for different structures and dimensions of an organization.

\subsection{Functional Requirements}

The functional requirements of the application can be divided in two groups. The former is regarding the operational implementation of functionalities that can be directly accessed by the users. The latter refers to functionalities that will help and guide users to access the previous functionalities according to their permission level and role performed in the system.

The first group of functionalities includes:

- Creation of users and ideas;

- Search for ideas based in date and hour (timestamp) in a descendent way;

- Search for inactive ideas;

- Activate/Inactivate users;

- Approve ideas.

The second group of features includes:

- Offer an interface to access the system to the user with two basic fields: "nickname" and "password";

- Restrict some operations in the system, according to the access level of each user.

The figure 1 gives a brief overview of all functionalities offered by the system using a user case UML diagram.

\footnotetext{
${ }^{1}$ http://crowdicity.com/en/

2 http://www.ideasmine.net/en/

3 http://www.ideacomb.com/
} 


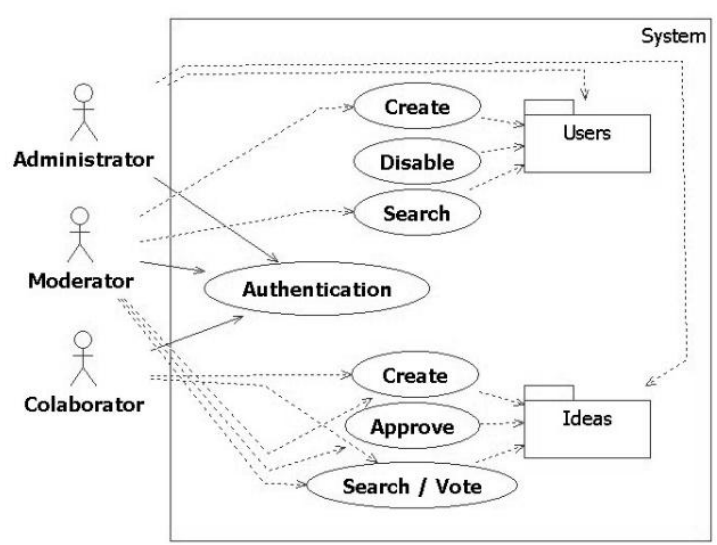

Fig 1. UML use cases diagram

The administrator has full control over the platform and its users, including permission to appoint another administrator and several other moderators. This feature guarantees that the platform will not be without any administration if the main administrator is unavailable. The moderator has the power to appoint other moderators, manage users and ideas. Finally, the user only has the possibility to publish an idea and vote for it.

The platform does not allow the modification and deletion of ideas previously submitted and approved in order to ensure the preservation of the original information provided by the user.

\subsection{Nonfunctional Requirements}

The non-functional requirements specify how the system should behave and it is typically responsible for the definition of the overall qualities or attributes of the application. It places restrictions on the product being developed, its process, and specifies external constraints that the product must meet.

The following non-functional requirements were established within the context of this application:

- Usability - it is the ease with which a user can learn to operate, prepare inputs for, and interpret outputs of a system or component;

- Performance - it concerns to the speed of operation of a system;

- Scalability - the graphic environmental is optimized to handle a wide variety of screen configuration sizes;

- Security - guarantee that unauthorized access to the system and its data is not allowed;

- Environmental - the application should be developed using interactive validation points in order to get validation and perform tests as soon as possible.

\subsection{Architecture of Application}

The system is structured to take advantage of open source technologies in order to provide a service with low cost operation and maintenance. The system architecture offers fixed and mobile clients access, a web page in PHP, a web server that supports PHP and a MySQL database. The architecture design took into account the expected high number of requests to the database from a future expansion of the project. Additionally, possible problems that could be arisen from large data volumes traded from different sources were taken in account. Therefore, the results of a query to the database will be minimized and optimized.
The figure 2 presents the architecture of the application.

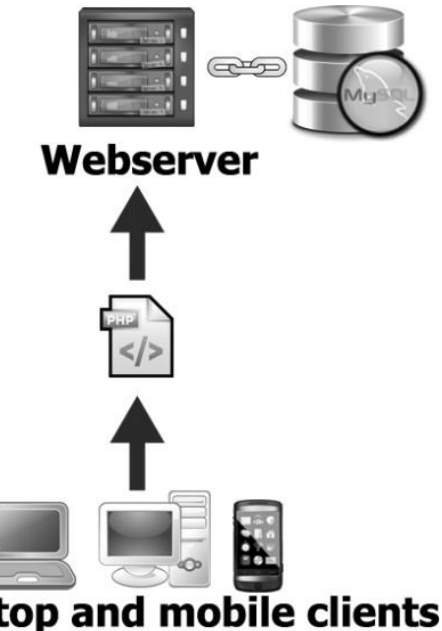

Fig 2. Architecture of the application

The server contains a MySQL database that is the core of the whole project. The database consists of four tables:

- Users table - contains personal data of all users of the system, as the "nickname", name, password, e-mail, and status, which can be enabled or disabled by the user;

- Roles table - represents the type of the user. Three types of roles were defined: administrators, moderators, and employees. Each role has specific permissions;

- Votes table - it is used to hold the vote of a user in a given idea. Furthermore, we saved the date and time of that assignment ("timestamp");

- Ideas table - it is adopted to store the innovative ideas of a user. It records a brief idea description in a text field, the state, the timestamp and the associated user.

The figure 3 represents the life cycle of an idea in the platform.

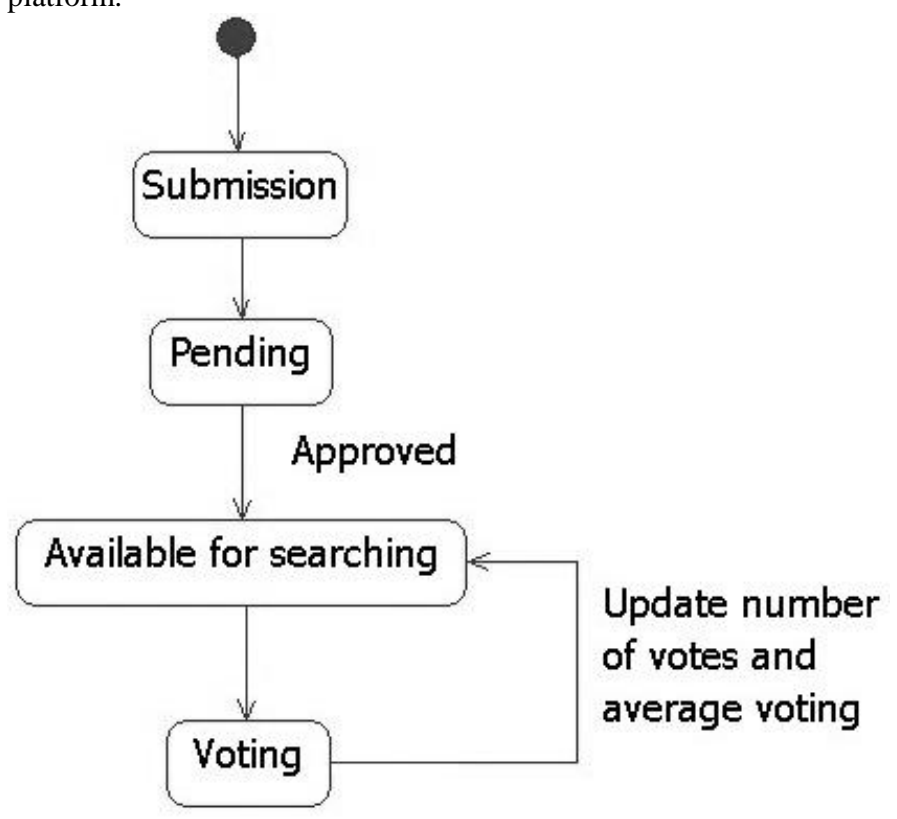

Fig 3. UML state case of an idea 
An idea has its starting point in the submission by a user of the platform, and then automatically follows to the pending state. At this stage, the idea awaits approval by an administrator or moderator. Once approved, the idea can be searched by the users and shall appear on the "home screen" of the platform. At his point, the user can click on an idea and vote according to the methodology of Likert. After voting, the number of votes and its corresponding average is updated, and the user is redirected to the main page, where he can check this information.

The web platform is accessible only to registered users. Twenty eight (28) validation and user acceptance tests were developed in order to confirm their functional and nonfunctional requirements.

\section{DISCUSSION}

The application was built in order to be simple and can be accessed by each company's employee from anywhere using a web browser, which avoid the necessity to install additional software and contributes significantly for the reduction in users' need for training and set-up costs. According to Franklya [21], the adoption of a web browser architecture contributes to the growth of interoperability, which is very useful for widely dispersed working groups. Furthermore, the application was built based exclusively in open source technologies in order to reduce investment needs and offer a platform-independent solution. Another important considered issue was the establishment of a great expansibility in order to support the future inclusion of new modules. Its modular structure turns the expansion of the application easier to get feedback from industry partners, customers and providers.

A central point that must be ensured is that all ideas are treated anonymously. The potential of each idea submitted by employees is evaluated by their peers, which improves the robustness and credibility of the considered evaluation process. The option to remain anonymous can make employees less nervous about submitting out-of-the-box ideas. According to Durmaz [22] staff may feel they can be more creative as they don't need to fear ridicule from their colleagues. However, at the same time, it is important that a senior manager thanks employees for their contribution or includes a reward process. Therefore, the program administrator knows the name of the person who submitted the idea. Nevertheless, in any situation, the employee remains anonymous to other employees.

Hassan [23] presents ten issues and advices how companies can improve their innovation programs. Among them we highlight four that can be mitigated by our application:

1. Irrelevant to customers or organizational needs - most suggestion schemes don't provide a model to understand customer outcomes and expectations. Additionally, idea boxes don't ensure alignment between the ideas and business strategy. Some ideas submitted may not be relevant for the company;

2. Too many ideas and poor processes - some ideas can be processed, others cannot. Over time, the suggestion box will have more ideas than can be managed by an innovation manager;

3. Lack of transparency - employees cannot see their ideas once they are in the box and do not know what is happening to their ideas. This can be demotivating for them;

4. Lack of oversight - in many cases, all the suggestions are processed by the same person, which may not always recognize the potential of a powerful idea particularly if it is outside of his/her area of expertise.

The considered approach allows and encourages the submission of ideas by all employees, some of them that work closely with customer or end-user, which will improve the quality level of ideas (issue 1). The existence of the moderator role that can be performed by different persons will remove a unique central congestion point (issue 2) and can be assigned attending to one's knowledge about the idea field (issue 4). Finally, employees can check the state of their ideas in the workflow process, which will help to motivate and encourage the participation (issue 3 ).

The application supports several desktop resolutions and was also tested in PDA devices and Apple iPhone and Samsung Galaxy mobile phones. Several tests were also performed in terms of robustness in order to avoid the existence of errors in the frontend layer, such as the introduction of alphanumeric characters in numeric fields, restriction in terms of characters for the idea description and establishment of standard grade for idea voting.

Several tests were made in order to test the page loading performance. These results are shown in figure 4 and figure 5 . The former illustrates a connection view (first view) scenario; the latter a connection view (repeat view) scenario. The orange line in both figures represents the CPU time and the green line represents the bandwidth allocation.

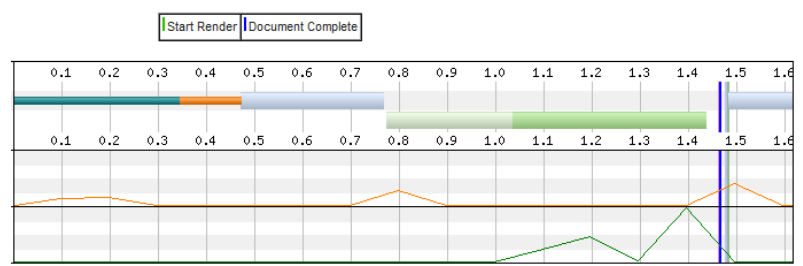

Fig 4. Connection view performance (first view)

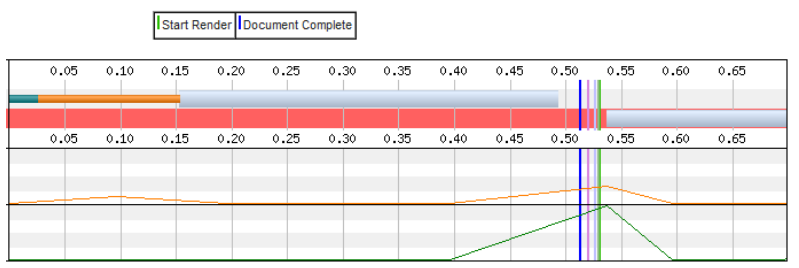

Fig 5. Connection view performance (repeat view)

In the first scenario the total loading page is around 1,464 seconds. This happens essentially because the web server needs to load all the style sheet files when it is invoked for the first time. In a second scenario, the total loading time decreases to around 0,523 seconds with the help of caching mechanisms.

The performance of the web application for mobile devices was also tested and provided a final score of 91/100. This score was obtained using the PageSpeed Insight provided by Google Developers. Some strength points includes the browser caching, prioritization of visible content, optimization of images, and minified CSS, HTML and Javascript. On the other hand, there are still some issues to be improved such as the elimination of render-blocking Javascript and CSS in above-the-fold content. 
Finally, it is relevant to mention that this application targets the segment of Small and Medium Enterprises (SMEs). Today's knowledge-based organizations depend on creativity for success, innovation, discovery and inventiveness. This also extends to small medium enterprises (SMEs) who, due to resource constraints and intense competition, depend on ideas in order to survive [24]. However, the majority of SMEs can be characterized as having a lack of available funds and resources when it comes to new services or product developments, which are under increasing pressure and financial strain, especially in the current economic climate, to deliver successful innovations. Therefore, the possibility to have a platform for idea management that is simultaneously simple to use, easily integrated and with low acquisition and maintenance costs, offers to SMEs the potential to foster collaboration among employees and increase their external competitiveness.

\section{CONCLUSIONS}

The preparation of this study will encourage a change in interpersonal relations through the promotion and dissemination of innovative ideas with potential within organizations. These organizations, in turn, benefit from a wider human capital as well as a chance to improve their everyday processes, reducing the time required for completion and any associated costs, eventually translating into a better chance of profits. In fact, the sharing of ideas on the platform and its view may even foster a better critical thinking skills of all participants, thus contributing to an organization evolve gradually, which may avoid the frequent use of external services audit.

At the technological level, the implementation of this project demonstrates that it is possible for an organization to base its operation in open source technologies, achieving greater productivity at a reduced or no cost. At social and business level, this application promotes greater equality of opinions giving the right credit of ownership to its author, avoiding the risk of usurpation of ideas by team leaders and management staff.

This prototype is still in an early development stage, but already demonstrates a high development potential and some market prospects. Future implementations are possible for the development of the platform, which will eventually become susceptible to be used daily and in a practical way for the user.

The main functionality to be implemented focuses on integration with social networks, such as: Facebook, Twitter, Pinterest and deviantART. The aim would be the creation of a plugin that allows the inclusion of a small button close to an image or text. So the user would have the ability to instantly submit an idea, avoiding authentication on the platform and keeping its line of though, without inducing any distraction in the preparation of the idea. A second feature would be the creation of a specific app for Android and iPhone, where the user could click on the application icon and submit immediately the desired text.

\section{REFERENCES}

[1] Nonaka, I. (1994). A dynamic theory of organizational knowledge creation. Organization science, 5(1), 14-37.

[2] Lindsey, J. S., Green, M. G., Murphy, J. T., Wood, K. L., \& Markman, A. B. (2005). Collaborating to success: An experimental study of group idea generation techniques. ASME Design Theory and Methodology Conference (pp 24-28).
[3] Butler, C. S. (2008). The very idea! A Corpus-based Comparison of IDEA, CONCEPT and NOTION and their Formal Equivalents in Spanish. Atlantis, 59-77.

[4] Godin, B. (2013). Invention, Diffusion and Linear Models of Innovation. Project on the Intellectual History of Innovation. Working paper $\mathrm{n}^{\circ} 15$. Retrieved at 12 April 2014 from http://www.chairefernanddumont.ucs.inrs.ca/wpcontent/uploads/2013/11/GodinB_2013_Invention_ Diffusion_and_Linear_Models_of_Innovation.pdf

[5] Gurteen, D. (1998). Knowledge, Creativity and Innovation. Journal of Knowledge Management, 2(1), 513.

[6] Shah, J. (1998). Experimental investigation of progressive idea generation techniques in engineering design. CD-ROM Proc. of the ASME DETC'98 (pp 1316).

[7] Shah, J. J., Kulkarni, S. V., \& Vargas-Hernandez, N. (2000). Evaluation of idea generation methods for conceptual design: effectiveness metrics and design of experiments. Journal of Mechanical Design, 122, 377.

[8] Jogalekar, U. A., \& Akhil Mangla. (2010). Idea generation algorithm based systems. 2010 3rd International Conference on Computer Science and Information Technology (pp 14-17).

[9] Goguen, J., (2009). What is a concept? Conceptual Structures: Common Semantics for Sharing Knowledge, 52-77.

[10] Munemori, J., Yoshino, T., \& Yunokuchi, K. (2001). A spiral-type idea generation method support system for sharing and reusing ideas among a group. 2001 IEEE International Conference on Systems, Man and Cybernetics. e-Systems and e-Man for Cybernetics in Cyberspace (Cat.No.01CH37236) (pp 1898-1903).

[11] Shigenobu, T., Yoshino, T., \& Munemori, J. (2003, January). Idea generation support system GUNGEN DX II beyond papers. In Knowledge-Based Intelligent Information and Engineering Systems (pp. 741-747). Springer Berlin Heidelberg.

[12] Camarinha-Matos, L., \& Afsarmanesh, H. (2006). Collaborative networks: Value creation in a knowledge society. Knowledge Enterprise: Intelligent Strategies in Product Design, Manufacturing, and Management. Proceedings of PROLAMAT 2006, IFIP TC5 International Conference, June 15-17, 2006, Shanghai, China (pp 26-40).

[13] Carneiro, L., Soares, A. L., Patrício, R., Alves, A., Madureira, R., \& de Sousa, J. P. (2007). Redes Colaborativas de Elevado Desempenho no norte de Portugal. Public Report. INESC Porto.

[14] Roth. C. (2005). Co-evolution in Epistemic NetworksReconstructing Social Complex Systems. Structure and Dynamics. Ph.D. dissertation.

[15] Pereira, C., Sousa, C., Soares, AL, (2012). Case studies on collaboration, technology and performance factors in business networks. International Journal of Computer Integrated Manufacturing, 26(11), 101-122. 
[16] Pereira, C. (2010). A Organização da Informação e Conhecimento em Redes Colaborativas como um Processo de Construção Social do Significado: Uma Teoria e um Método Prático, Ph.D. dissertation, Faculdade de Engenharia Universidade do Porto.

[17] Hautz, J., Hutter, K., Fuller, J., Matzler, K., \& Rieger, M. (2010). How to Establish an Online Innovation Community? The Role of Users and Their Innovative Content. 2010 43rd Hawaii International Conference on System Sciences (pp 1-11).

[18] Vara, Hugo Jorge Cavaleiro (2008). Creativity and Idea Management in Collaborative Networks: The Role of Information Management, MSc. Thesis, Faculdade de Engenharia Universidade do Porto.

[19] Björk, J., \& Magnusson, M. (2009). Where Do Good Innovation Ideas Come From? Exploring the Influence of Network Connectivity on Innovation Idea Quality. Journal of Product Innovation Management, 26(6), 662670.
[20] Fritsch, M., \& Kauffeld-Monz, M. (2008). The impact of network structure on knowledge transfer: an application of social network analysis in the context of regional innovation networks. The Annals of Regional Science, 44(1), 21-38.

[21] Franklya, C. (2010). Computer Supported Cooperative Work (CSCW(: Some Comments. Journal of Language, Culture and Communication (JLCC), 7(8), 20-28.

[22] Durmaz, L. (2013). How to Create an Employee Suggestion Form. Available on http://imblog.ideaglow.com/how-to-create-employeesuggestion-form/ (accessed on 28th June 2014).

[23] Hassan, K. (2010). 10 Reasons Why Innovation Suggestion Schemes and Idea Boxes Fail. Available on http://www.innovationexcellence.com/blog/2010/06/06/1 0 -reasons-why-innovation-suggestion-schemes-and-ideaboxes-fail/ (accessed on 2nd July 2014).

[24] Perkins, G. (2014). Factors External to the Individual Encouraging Idea Generation in SME Contexts. Plymouth University. Ph.D. dissertation. 